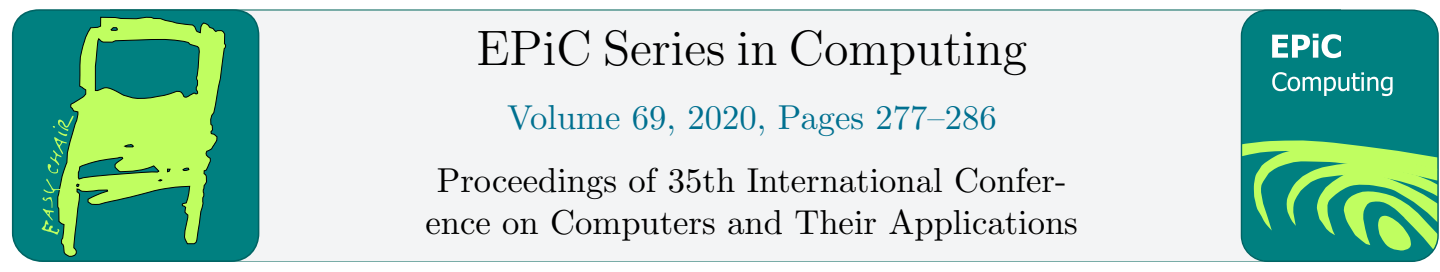

\title{
Productivity Comparison among Software Projects
}

\author{
Cuauhtémoc López-Martín \\ Universidad de Guadalajara, Jalisco, México \\ cuauhtemocecucea.udg. mx
}

\begin{abstract}
Software engineering management (SEM) involves as activities to planning, coordinating, measuring, monitoring, and controlling. Since maximizing productivity is related to the highest value with lowest resource consumption, a factor taken into account in these activities is productivity, which includes total effort used to satisfy the exit criteria of a software process. In recent years, productivity has been studied from several points of view; therefore, the contribution of this study is analyze its variability by classifying the software projects based on their size measure, type of development, development platform, and programming language type such that the SEM activities are more objective. In this study, data sets of software projects were selected from the International Software Benchmarking Standards Group Release 2018 for performing the following three experiments between types of development, and by type of size measure: (1) independent of both the type of development platform and of the programming language type, (2) dependent of the type of development platform, and independent of the programming language type, and (3) dependent of both the type of development platform and of the programming language type. Results show the statistically significant differences by experiment.
\end{abstract}

\section{Introduction}

In the software engineering field, the software productivity (SP) measurement is needed to control and improve the performance of software development [1]. A survey published in 2014 revealed that SP represented a concern for software engineers in the sense that they would like scientists to investigate [2]. Since 2014, SP has been analyzed from a several points of view [3] [4] [5] [6] [7] [8] [9] [10]; however, I did not find any study whose objective has been to statistically analyze the SP taking into account the type of size measure; type of development (TD) such as new or enhancement; development platform (DP) such as Mainframe, MidRange, Multiplatform, and Personal Computer; and programming language type (PLT) classified from its generation, thus, this study contributes by answering the following question: 
Is there statistical difference between the software productivity of separated project data sets classified from their type of size measure, TD, DP, and PLT, or the data sets should be used without that these criteria are taken into account (i.e., using pooled data sets)?

The answer to this question will be useful to software engineers such that they correctly select software projects to either analyze or compare the SP between types of projects, as well as to generate SP prediction models.

SP can be analyzed from abstraction levels such as organization, project, individual, and task [1]. The answer to the previous question is related to the analysis of projects selected from the International Software Benchmarking Standards Group (ISBSG) Release 2018, which is an international public repository of software projects [11].

SP is calculated using the ratio of output to input for each measured output primitive for which related input effort data is collected [12]. The measured output has commonly been based on either source lines of code or functional size measure (FSM) [13]. There are several types of FSM such as IFPUG, MARK II, NESMA, Fisma, and COSMIC [14].

In the ISBSG, the project SP delivery rate is reported in hours by FSM unit: it is calculated from work effort (i.e., person-hours) for the development team divided by FSM [14].

In this study, the following three experiments are performed between types of development and by type of FSM:

- Independent of (1) the type of development platform and (2) the programming language type;

- Dependent of the type of development platform, and independent of the programming language type;

- Dependent (1) of the type of development platform and (2) of the programming language type. The hypotheses to be tested are the following:

$H_{l}$ : There is statistically difference of the productivity between new and enhancement software projects independent of the (1) type of development platform and (2) programming language type

$\mathrm{H}_{2}$ : There is statistically difference of the productivity between software projects dependent of the type of development platform, and independent of the programming language type

$H_{3}$ : There is statistically difference of the productivity between software projects dependent of the (1) type of development platform and (2) programming language type

The rest of this study is organized as follows: Section 2 mentions recent studies related to SP. Section 3 describes the criteria to select the data sets from the ISBSG used in this study. Section 4 presents the statistical analysis on SP data sets. Section 5 shows the results of Section 4, whereas Section 6 presents the conclusions, the limitations, validation threat, and future work of this study.

\section{Related work}

SP has recently been studied from points of view such as the relationship among quality maturity levels and labor productivity (2017) [10], how the project managers perceive the productivity of developers (2016) [4], SP prediction models (2016) [9], the influence of types programming languages on SP (2016) [3], relationship between size and productivity of teams of developers (2016) [5], social aspects by analyzing the impact of selected team-based variables over the latent constructs of productivity (2015) [15], the analysis of affects such as emotions, moods, and feelings, that have a linkage to cognitive processing activities and the productivity of software developers (2015) [6], how project duration, upfront costs and uncertainty interact and impact on SP (2015) [8], and SP improvement in global software development [7] (2015). A systematic study on SP involving 38 studies 
[1] suggests documenting the context when comparing productivities, therefore, in the present study, the SP is statistically analyzed taking into account attributes of software projects such as project size type, TD, DP, and PLT.

\section{Description of data sets}

The ISBSG release 2018 is an international public repository of software projects developed between 1989 and 2016. It contains 8,261 projects developed in 32 different countries such as Spain, which reports the $18 \%$ of that 8,261 , United States (14.5\%), Netherlands (13\%), Finland $(10.8 \%)$, France (8.5\%), Australia (7.4\%), India (6.9\%), Japan (5\%), Canada (3.7\%), and Denmark with 3,2\% [11]. Table 1 describes the number of projects by applying each criterion suggested by the ISBSG [16]. Regarding quality rating, the ISBSG classifies the projects from "A" to "D". Since the ISBSG suggests to the "A" and "B" classifications for statistical analysis [16], these two types were only used as criteria for selecting the data sets for the present study.

As for functional sizing methods (FSM), three of them had the major number of software projects: IFPUG 4+, NESMA, and Fisma. In accordance with ISBSG guidelines, IFPUG V4 projects with V4 and post V4 should not be mixed. In addition, NESMA can be mixed with IFPUG 4+ [16]. Table 2 includes those final 2,908 of Table 1 classified by FSM type, and type of development.

\begin{tabular}{lll}
\hline \multicolumn{1}{c}{ Attribute } & \multicolumn{1}{c}{ Selected value(s) } & Projects \\
\hline Productivity delivery rate not null & --- & 6,476 \\
Data quality rating & A, B & 6,133 \\
Unadjusted Function Point Rating & A, B & 5,710 \\
Development platform not null & --- & 3,370 \\
Programming language type not null & --- & 3,082 \\
Functional sizing methods & IFPUG 4+, NESMA, Fisma & 2,908 \\
\hline
\end{tabular}

Table 1: Criteria for selecting the data sets from the ISBSG $(8,261$ projects)

\begin{tabular}{llr}
\hline \multicolumn{1}{c}{ FSM } & \multicolumn{1}{c}{ TD } & Projects \\
\hline Fisma & New & 275 \\
\multirow{2}{*}{ IFPUG 4+, NESMA } & 234 \\
& Enhancement & 748 \\
& New & 1,619 \\
& Enhancement & 32 \\
& Re-development & 2,908 \\
\hline
\end{tabular}

Table 2: Projects classified by functional sizing method (FSM) and type of development (TD)

\section{Statistical Productivity Analysis}

The three experiments mentioned in the Introduction section of this study will be performed by FSM type. Statistical tests are selected in accordance with the number of groups (data sets) to be compared, data dependence, and data distribution [17]. The latter one is performed by applying the Chi-squared $\left(\chi^{2}\right)$, Shapiro-Wilk, skewness, and kurtosis statistical tests [17]. In the following sections, each experiment is described. 


\subsection{First experiment}

Description: Independent of (1) the type of development platform and (2) of the programming language type.

a) Number of groups to be compared: two (new and enhancement)

b) Data dependence: software projects were independently developed, therefore, data are independent.

\section{Fisma FSM:}

c) Data distribution: Since the 275 new and 234 enhancement projects of Table 2 are independent, the 509 projects of these two data sets were pooled to apply the normal statistical tests. Each of the four $\mathrm{p}$-values had a value lower than 0.01 , therefore, the notion that the data comes from a normal distribution can be rejected with $99 \%$ confidence.

Since two data sets are compared (i.e., new and enhancement ones), they are independent, and the data are not normality distributed, the suitable statistical test to compare the data sets is Mann-Whitney $\mathrm{W}$ [17], which tests the null hypothesis that the medians within each of the data sets is the same. The productivity median values were 6.3 and 7.45 for new and enhancement projects, respectively. The pvalue for this test was equal to 0.0007 , thus, there is a statistically significant difference amongst the medians of the two data sets at the $99.0 \%$ confidence level.

\section{IFPUG V4+ and NESMA FSM:}

d) Data distribution: Since the 748 new and 1619 enhancement projects of Table 2 are independent, the 2,367 projects of the two data sets were pooled to apply the normal statistical tests. Their four p-values were lower than 0.01 , therefore, the notion that the data comes from a normal distribution can be rejected with $99 \%$ confidence.

Based on these results, the statistical test to be applied is Mann-Whitney W [17]. The productivity median values were 8.7 and 13 for new and enhancement projects, respectively. The p-value for this test was lower than 0.01 , that is, there is a statistically significant difference amongst the medians of the two data sets at the $99 \%$ confidence level.

\subsection{Second experiment}

Description: Dependent of the type of development platform, and independent of the programming language type. Table 3 includes the mean and median by data set.

\section{Fisma FSM:}

Among types of DPs by TD:

New projects: The four new FSM data sets of Table 3 were pooled to apply the four normality tests. The results in their four tests were lower than 0.01 , then, the hypothesis of that the data set of 275 projects has a normal distribution was rejected at $99 \%$ confidence. Thus, the Kruskal-Wallis test is applied [17], which tests the null hypothesis that the medians within each of the four types of DP are the same. The p-value Kruskal-Wallis was equal to 0.0044 , that is, there is a statistically significant difference amongst the medians at the $99 \%$ confidence level. To determine which medians are significantly different from which others, an analysis by pair was performed. A new set of differences of ARs by pair was generated and its normal distribution was analyzed. If at least one of the four pvalues by pair of Table 4 was lower than 0.01 , then the notion that the data comes from a normal distribution can be rejected with $99 \%$ confidence. Table 4 shows that the six cases do not present a normal distribution, thus, a Mann-Whitney W statistical test was performed to compare each pair of data sets.

Enhancement projects: The four enhancement data sets of Table 3 were pooled to apply the four normality tests, the results for the four tests were lower than 0.01 , then, the hypothesis of that the data set of 234 projects has a normal distribution was rejected. The p-value Kruskal-Wallis was equal to 
0.0845 , then, there is not a statistically significant difference amongst the medians at the $95 \%$ confidence level.

\begin{tabular}{lllrrr}
\hline \multicolumn{1}{c}{ FSM } & \multicolumn{1}{c}{ TD } & DP & NP & Mean & Median \\
\hline Fisma & New & MF & 86 & 7.8 & 7.0 \\
& & MR & 35 & 8.4 & 7.3 \\
& & Multi & 97 & 7.5 & 6.3 \\
& \multirow{4}{*}{ Enhancement } & PC & 57 & 6.0 & 4.3 \\
& & MF & 138 & 9.4 & 7.8 \\
& & MR & 19 & 10.6 & 7.3 \\
& & Multi & 69 & 7.6 & 7.1 \\
IFPUGV4+, NESMA & \multirow{2}{*}{ New } & PC & 8 & 5.5 & 5.2 \\
& & MF & 204 & 21.8 & 15.7 \\
& & MR & 76 & 13.9 & 8.7 \\
& & Multi & 223 & 11.9 & 7.3 \\
& \multirow{2}{*}{ Enhancement } & PC & 245 & 12.5 & 7.0 \\
& & MF & 646 & 22.6 & 14.4 \\
& & MR & 124 & 18.9 & 13.7 \\
& & Multi & 648 & 17.2 & 13.1 \\
& & PC & 201 & 11.1 & 7.6 \\
\hline
\end{tabular}

Table 3: Central tendency measures for data sets

\begin{tabular}{lccccc}
\hline \multirow{2}{*}{ DPs } & \multicolumn{4}{c}{ Normality test (data of two sets are pooled) } & \multirow{2}{*}{$\begin{array}{c}\text { Mann-Whitney W } \\
\text { p-value }\end{array}$} \\
\cline { 2 - 5 } & $\chi^{2}$ & S-W & Skewness & Kurtosis & 0.8302 \\
MF - MR & 0.0038 & 0.0000 & 0.0000 & 0.0000 & 0.4344 \\
MF - Multi & 0.0000 & 0.0000 & 0.0000 & 0.0002 & 0.0007 \\
MF - PC & 0.0000 & 0.0000 & 0.0000 & 0.0000 & 0.4977 \\
MR - Multi & 0.0006 & 0.0000 & 0.0000 & 0.0000 & 0.0091 \\
MR - PC & 0.0000 & 0.0000 & 0.0001 & 0.0024 & 0.0064 \\
Multi - PC & 0.0000 & 0.0000 & 0.0000 & 0.0000 & \\
\hline
\end{tabular}

Table 4: Statistical analysis by pair of new data set projects (Fisma FSM)

\section{Between TDs by DP:}

Four pooled sets of 224, 54, 166, and 65 projects by DP were integrated to apply the four normality tests. Their four tests were lower than 0.01 , then the hypothesis of that the each data set has a normal distribution was rejected at the 99\% confidence level. Table 6 shows that the four p-values corresponding to the Mann-Whitney W test for Fisma were higher than 0.05, then, there is not a statistically significant difference between the medians at the $95 \%$ confidence level.

IFPUG V4+ and NESMA FSM:

Among types of DPs by TD.

New projects: The four new data sets of Table 3 were pooled to apply the four normality tests, the results in their four tests were lower than 0.01 , then, the hypothesis of that the data set of 748 projects has a normal distribution was rejected at the $99 \%$ confidence. Thus, the Kruskal-Wallis test was applied, whose p-value was equal to 0.0000 , that is, there is a statistically significant difference amongst the medians at the $99 \%$ confidence level. Since results of normality tests showed that none pair has normal distribution, an analysis applying the Mann-Whitney W test is performed by pair in Table 5 .

Enhancement projects: The four enhancement data sets were pooled to apply the four normality tests. The results for four tests were lower than 0.01 , then, the hypothesis of that the data set of 1,619 projects has a normal distribution was rejected. The p-value Kruskal-Wallis was equal to 0.0000 , that is, there is a statistically significant difference amongst the medians at the $99 \%$ confidence level. To 
determine which medians are significantly different from which others, an analysis by pair is performed in "Enhancement" column of Table 5.

\begin{tabular}{lcc}
\hline \multirow{2}{*}{ DPs } & \multicolumn{2}{c}{ Mann-Whitney W p-value } \\
\cline { 2 - 3 } & New & Enhancement \\
\hline MF - MR & 0.0013 & 0.2488 \\
MF - Multi & 0.0000 & 0.0169 \\
MF - PC & 0.0000 & 0.0000 \\
MR - Multi & 0.0686 & 0.9392 \\
MR - PC & 0.0077 & 0.0000 \\
Multi - PC & 0.0930 & 0.0000 \\
\hline
\end{tabular}

Table 5: Statistical analysis by pair of data sets from their TD (IFPUGv4+ and NESMA FSM)

\section{Between types of TDs by DP:}

Four pooled sets of $850,200,871$, and 446 projects by DP were integrated to apply the four normality tests. Their four tests were lower than 0.01 , then, the hypothesis of that the each data set has a normal distribution was rejected at the 99\% confidence level. Table 6 shows that two p-values corresponding to the Mann-Whitney W test were lower than 0.05 , then, there is statistically significant difference between the medians of the MR, as well as Multi at the $95 \%$ confidence level.

\begin{tabular}{lcc}
\hline \multirow{2}{*}{ DP } & \multicolumn{2}{c}{ Mann-Whitney W p-value } \\
\cline { 2 - 3 } & Fisma & IFPUGv4+ and NESMA \\
\hline MF & 0.0790 & 0.8763 \\
MR & 0.4253 & 0.0228 \\
Multi & 0.4649 & 0.0000 \\
PC & 0.6105 & 0.2871 \\
\hline
\end{tabular}

Table 6: Statistical analysis by pair of data sets between types of TDs by DP

\subsection{Third experiment}

Description: Dependent of (1) the type of development platform and (2) the programming language type. Since kurtosis and $\chi^{2}$ statistical distribution tests need at least 20 and 30 software projects, respectively, only those data sets having thirty or more projects were selected to this experiment. Table 7 includes those selected data sets to be compared. Each data set was not normally distributed, thus, a Kruskal-Wallis test was performed. The p-values of Table 7 mean that there was statistically difference at $99 \%$ confidence for five cases, and at $95 \%$ for one case. Therefore, Table 8 shows those pairs (eighteen) whose results after applying a Mann-Whitney W test presented statistically significant difference. This statistical test was selected because each new data set generated from the differences between ARs of the two data sets to be compared, was non-normally distributed.

\begin{tabular}{|c|c|c|c|c|c|c|c|c|c|c|c|}
\hline \multirow[t]{2}{*}{ FSM } & \multirow[t]{2}{*}{ TD } & \multirow[t]{2}{*}{ PLT } & \multicolumn{8}{|c|}{ DP } & \multirow{2}{*}{$\begin{array}{c}\text { Kruskal- } \\
\text { Wallis }\end{array}$} \\
\hline & & & MF & Median & MR & Median & Multi & Median & $\mathrm{PC}$ & Median & \\
\hline \multirow[t]{2}{*}{ Fisma } & New & $3 \mathrm{GL}$ & 78 & 7.00 & --- & --- & 72 & 6.35 & 45 & 4.50 & 0.0158 \\
\hline & Enhancement & 3GL & 119 & 7.70 & --- & --- & 38 & 6.30 & --- & --- & 0.0093 \\
\hline \multirow{4}{*}{$\begin{array}{c}\text { IFPUGV4+, } \\
\text { NESMA }\end{array}$} & New & 3GL & 151 & 17.80 & 42 & 9.30 & 110 & 11.15 & 82 & 10.10 & 0.0005 \\
\hline & & 4GL & 39 & 7.40 & 33 & 8.20 & 113 & 5.40 & 161 & 5.50 & 0.0073 \\
\hline & Enhancement & 3GL & 516 & 15.40 & 70 & 14.45 & 446 & 14.05 & 101 & 9.30 & 0.0000 \\
\hline & & $4 \mathrm{GL}$ & 62 & 7.60 & 54 & 12.85 & 202 & 11.60 & 88 & 7.00 & 0.0000 \\
\hline
\end{tabular}

Table 7: Data sets dependent of the type of development platform, and of the programming language type 


\begin{tabular}{ccll}
\hline FSM & TD & PLT & \multicolumn{1}{c}{ Pairs of DPs } \\
\hline Fisma & New & 3GL & MF - PC, Multi - PC \\
& Enhancement & 3GL & MF - Multi \\
& New & 3GL & MF - MR, MF - Multi, MF - PC \\
& & 4GL & MF - Multi, MF - PC, MR - Multi, MR - PC \\
& \multirow{2}{*}{ Enhancement } & 3GL & MF - Multi, MF - PC, MR - PC, Multi - PC \\
& & 4GL & MF - MR, MF - Multi, MR - PC, Multi - PC \\
\hline
\end{tabular}

Table 8: Pairs of data sets by PLT from Table 7 having statistical significance

\section{Results}

In this section, those accepted hypotheses formulated by experiment in the Introduction section are mentioned. They are written in accordance with the results obtained in Section 4.

\section{a) First experiment}

The median values for Fisma FSM were 6.3 and 7.45 for new and enhancement projects, respectively, whereas for IFPUG 4+ and NESMA were 8.7 and 13 for new and enhancement projects, respectively. In this experiment, the following hypothesis was accepted at the $99 \%$ confidence level for both types of FSM (i.e, Fisma, and IFPUG 4+ and NESMA):

$H_{1}$ : There is statistically difference of the productivity between new and enhancement software projects independent of the type of development platform, and of the programming language type

\section{b) Second experiment}

\section{b.1) Fisma FSM}

The median values for MF, MR, Multi and PC for new projects were 7.0, 7.3, 6.3, and 4.3 respectively. The following hypothesis was accepted at the $99 \%$ confidence level

$\mathrm{H}_{2}$ : There is statistically difference of the productivity between new software projects dependent of the type of development platform, and independent of the programming language type between MF and PC, MR and PC, and Multi and PC.

As for enhancement projects, the median values for MF, MR, Multi and PC were 7.8, 7.3, 7.1, and 5.2 respectively, and there was not statistically significant difference amongst these medians at the $95 \%$ confidence level.

In accordance with TDs by DP, there was not statistically significant difference for any of the four comparisons among their medians at the $95 \%$ confidence level.

\section{b.2) IFPUGV4+ and NESMA FSM}

The median values for MF, MR, Multi and PC for new projects were 15.7, 8.7, 7.3, and 7.0 respectively. The following hypothesis was accepted at the $99.0 \%$ confidence level

$\mathrm{H}_{2}$ : There is statistically difference of the productivity between new software projects dependent of the type of development platform, and independent of the programming language type between MF and MR, MF and Multi, MF and PC, and MR and PC.

The median values for MF, MR, Multi and PC for enhancement projects were 14.4, 13.7, 13.1, and 7.6 respectively. The following hypothesis was accepted at the $95.0 \%$ confidence level

$\mathrm{H}_{2}$ : There is statistically difference of the productivity between enhancement software projects dependent of the type of development platform, and independent of the programming language type between MF and Multi, MF and PC, MR and PC, and Multi and PC. 
Regarding TDs by DP, there was statistically significant difference between the medians of the MR, as well as Multi level at the $95 \%$ confidence level.

c) Third experiment

The following hypothesis was accepted for those eighteen cases included in Table 10 whose p-value was lower or equal to 0.05 .

$H_{3}$ : There is statistically difference of the productivity between software projects dependent of the type of development platform and programming language type

\section{Conclusions}

A software engineering survey consisting of 145 questions was published in 2014. It asked questions that software engineers would like scientists to investigate, and thirteen of those 145 questions was related to SP. The SP measurement is necessary either to control or to improve the performance of software development. Therefore, in this study, a statistical analysis on software projects selected taking into account their type of size measure, TD, DP, and PLT was proposed with the objective of that practitioners and researchers correctly perform a selection of software projects to either analyze or compare the SP between types of projects, as well as for generating models to predict SP.

After the results presented in Section 5, the following conclusions can be written by experiment:

\section{a) First experiment}

There was statistical difference of the productivity between new and enhancement software projects independently of their type of development platform, and of their programming language type for the two types of FSM (i.e, Fisma, and IFPUG 4+, NESMA). The productivity of enhancement projects was higher than the productivity of new projects for the two types of FSM.

\section{b) Second experiment}

b.1) Fisma FSM

There was statistical difference of the productivity between new software projects dependent of their type of development platform, and independently of the programming language type between MF and PC, MR and PC, and Multi and PC. The productivity values for projects developed on PC were lower than those ones of MF, MR and Multi.

Regarding enhancement projects, there was not significant difference amongst the productivity of the four types of development platforms.

As for TDs by DP comparison, there was not statistically significant difference among the productivity of the four types de types of developments.

b.2) IFPUGV4+ and NESMA FSM

There was statistical difference of the productivity between new software projects dependent of their type of development platform, and independently of the programming language type between MF and MR, MF and Multi, MF and PC, and MR and PC. The productivity values for projects developed on MF were higher than those ones of MR, Multi, and PC, whereas that of MR was higher than that of PC.

In accordance with enhancement projects, there was significant difference between the productivity of MF and Multi, MF and PC, MR and PC, and Multi and PC. MF presented the highest productivity value among them, $\mathrm{PC}$ the lower one, whereas MR had a value higher that Multi.

Regarding TDs by DP, there was statistically significant difference between the productivity between MR, as well as Multiplatform data sets.

\section{c) Third experiment}

There were eighteen pairs (Table 8) showing difference in their productivity taking into account type of development, type of development platform, and programming language type (Table 8).

Based on these results and from a practical point of view, the software manager having data obtained from his organization whose software projects have been developed on different development platforms 
and coded in programming language generations can (1) statistically analyze his data sets to conclude if they could be pooled, or (2) use data of the projects used in the present study as a reference, such that the manager generates prediction models on productivity.

A limitation of the present study is that after applying the criteria suggested for the ISBSG to select projects (Tables 1 and 2), I could only analyze some data sets.

A validation threat of this study is that each FSM value is calculated by the practitioner or researcher from software features, therefore, the results of this study also depends of that calculation reported to the ISBSG.

Future work will be related to the proposal of models for the new or enhancement software projects analyzed in this study. The types of models to be proposed will be classifiers and prediction models. In accordance with the productivity value, a software project could be classified in very low, low, medium, or high; whereas regarding prediction models, statistical regression and machine learning models can be proposed to predict SP. Classifiers will be based on Bidirectional Associative Memories [18], ontologies [19], Alpha-Beta models [20], and associative models [21], whereas models such as [22] [23] [24] and [24] will be applied for SP prediction.

\section{Acknowledgement}

The author would like to thank CUCEA of Universidad de Guadalajara, Jalisco, México, Programa para el Desarrollo Profesional Docente (PRODEP), as well as to Consejo Nacional de Ciencia y Tecnología (CONACYT).

\section{References}

[1] K. Petersen, "Measuring and predicting software productivity: A systematic map and review," Information and Software Technology, Elsevier, vol. 53, no. 4, 2011, pp. 317-343

[2] A. Begel and T. Zimmermann, "Analyze this! 145 questions for data scientists in software engineering" in Proceedings of the 36th International Conference on Software Engineering, Hyderabad, 2014.

[3] L. Lavazza, S. Morasca and D. Tosi, "An empirical study on the effect of programming languages on productivity," in Proceedings of the 31st Annual ACM Symposium on Applied Computing, Pisa, Italy, 2016.

[4] E. Oliveira, T. Conte, M. Cristo and E. Mendes, "Software Project Managers' Perceptions of Productivity Factors: Findings from a Qualitative Study," in Proceedings of the 10th ACM/IEEE International Symposium on Empirical Software Engineering and Measurement, Ciudad Real, Spain, 2016.

[5] I. Scholtes, P. Mavrodiev and F. Schweitzer, "From Aristotle to Ringelmann: a large-scale analysis of team productivity and coordination in Open Source Software projects," Empirical Software Engineering, Springer, vol. 21, 2016, pp. 642-683

[6] D. Graziotin, X. Wang and P. Abrahamsson, "Do feelings matter? On the correlation of affects and the selfassessed productivity in software engineering," Journal of Software: Evolution And Process, Wiley, vol. 27, no. 7, 2015, pp. 467-487.

[7] D. Manoj Ray and P. Samuel, "Improving the productivity in global software development," in Proceedings of the 6th International Conference on Innovations in Bio-inspired Computing and Applications, Kochi, India, 2015.

[8] L. L. Xiaoying Kong and J. Chen, "How project duration, upfront costs and uncertainty interact and impact on software development productivity? A simulation approach," International Journal of Agile Systems and Management, vol. 8, no. 1, 2015, pp. 39-52 
[9] J. Wu and S. Gao, "Software Productivity Estimation by Regression and Naïve-Bayes Classifier. An Empirical Research," in Proceedings of the ICPIT 2016 The International Conference on Promotion of Information Technology, Banff, 2016.

[10] C. H. C. Duarte, "Productivity paradoxes revisited," Empirical Software Engineering, Springer, vol. 22 , no. 2 , 2017, p. 818-847

[11] ISBSG, "ISBSG Demographics", International Software Benchmarking Standards Group, 2018.

[12] C/S2ESC - Software \& Systems Engineering Standards Committee, "1045-1992 - IEEE Standard for Software Productivity Metrics," IEEE Standards Association, 2002.

[13] Sheetz, S.D., Henderson, D., Wallace, L., "Understanding developer and manager perceptions of function points and source lines of code“. The Journal of Systems and Software, Elsevier, vol. 82, 2009, 1540-1549. Doi:10.1016/j.jss.2009.04.038

[14] ISBSG, "Field Descriptions ISBSG D\&E Repository, Release 2018", International Software Benchmarking Standards Group, 2018.

[15] M. Yilmaz, R. O'Connor and P. Clarke, "Effective Social Productivity Measurements during Software Development - An Empirical Study," International Journal of Software Engineering and Knowledge Engineering, vol. 26, no. 3, 2015, pp. 457-490.

[16] ISBSG, "Guidelines for use of the ISBSG data, Release 2018", International Software Benchmarking Standards Group, 2018.

[17] C. López-Martín, "Predictive Accuracy Comparison between Neural Networks and Statistical Regression for Development Effort of Software Projects", Applied Soft Computing, Elsevier, vol. 27, 2015, pp. 434-449. DOI: 10.1016/j.asoc.2014.10.033

[18] M.E. Acevedo, C. Yáñez-Márquez, M.A. Acevedo, "Bidirectional Associative Memories: Different Approaches", ACM Computing Surveys, Association for Computing Machinery, Vol. 45, No. 2, 2013, pp. 1-30. DOI:10.1145/2431211.2431217

[19] S. Cerón-Figueroa, I. López-Yáñez, W. Alhalabi, O. Camacho-Nieto, Y. Villuendas-Rey, M. Aldape-Pérez, C. Yáñez-Márquez, "Instance-based ontology matching for e-learning material using an associative pattern classifier", Computers in Human Behavior, Elsevier, Vol. 69, 2017, pp. 218225. DOI:10.1016/j.chb.2016.12.039

[20] López-Yáñez, I., Yáñez-Márquez, Cornelio, Camacho-Nieto, O., Aldape-Pérez, M., ArgüellesCruz, A.J. "Collaborative learning in postgraduate level courses", Computers in Human Behavior, Elsevier, Vol. 51, Part B, 2015, pp. 938-944. DO:10.1016/j.chb.2014.11.055

[21] I. López-Yáñez, L. Sheremetov, C. Yáñez-Márquez, “A Novel Associative Model for Time Series Data Mining", Pattern Recognition Letters, Elsevier, Vol. 41, 2014, pp. 23-33. DOI:10.1016/j.patrec.2013.11.008

[22] J.A. Antón-Vargas, Y. Villuendas-Rey, C. Yáñez-Márquez, I. López-Yáñez, O. Camacho-Nieto, "Improving the performance of an associative classifier by Gamma Rough Sets based instance selection", International Journal of Pattern Recognition and Artificial Intelligence, World Scientific, Vol. 32, No. 1, 2018, pp. 1860009. DOI:10.1142/S0218001418600091

[23] A.V. Uriarte-Arcia, C. Yáñez-Márquez, J. Gama, J., I. López-Yáñez, O. Camacho-Nieto, “Data Stream Classification Based on The Gamma Classifier", Mathematical Problems in Engineering, Hindawi, Vol. 2015, Article 939175, 17 pages. DOI:10.1155/2015/939175

[24] C. Yáñez-Márquez, I. López-Yáñez, M. Aldape-Pérez, O. Camacho-Nieto, Y. Villuendas-Rey, "Theoretical Foundations for the Alpha-Beta Associative Memories: 10 Years of Derived Extensions, Models, and Applications", Neural Processing Letters, Springer, Vol. 48, Issue 2, 2018, pp. 811-847. DOI:https://doi.org/10.1007/s11063-017-9768-2

[25] I. López-Yáñez, A.J. Argüelles-Cruz, O. Camacho-Nieto, C. Yáñez-Márquez, "Pollutants time series prediction using the Gamma classifier", International Journal of Computational Intelligence Systems, Atlantis Press, Vol. 4, Issue 4, 2011, pp. 680-711. DOI:10.2991/ijcis.2011.4.4.23 